\title{
Remarks on triples in enriched categories
}

\section{H. Wiesler and G. Calugareanu ir}

\begin{abstract}
Let $V$ be a symetric monoidal closed category with equalizers.
The V-triples $T, T^{\prime}, \ldots$ in the enriched category $A$, together with suitably defined morphisms form a category $V$-Trip $(A)$. The V-categories $A^{\top}, A^{T^{\prime}}, \ldots$ and the V-functors $R: A^{T^{\prime}} \rightarrow A^{\top}$ which are compatible with the forgetful functors form a category $V_{-A l g}(A)$. In the subsequent note it is shown that $V$ - Trip(A) is isomorphic to the dual of $V_{-A l g}(A)$ and that the morphisms of $V$-Alg $(A)$ are inverse limit preserving $V$-functors.
\end{abstract}

\section{0 . Introduction}

In [5], Frei considers the category of the triples in a category $A$, and of the triple morphisms, $\operatorname{Trip}(A)$, and the category of the categories of algebras $A^{\top}, A^{T^{\prime}}, \ldots$ and of the functors which are compatible with the forgetful functors, $\mathrm{Alg}(A)$. He shows that $\operatorname{Tr} i p(A)$ is isomorphic to the dual of $\mathrm{Alg}(A)$ and that the morphisms of $\mathrm{Alg}(A)$ preserve (inverse) limits.

Let $U$ be a symetric monoidal closed category, in the Eilenberg-Kelly [3] sense. In [3] the $V$-categories, which are also called enriched categories in Day-Kelly [2], are constructed. The $V$-triples in $V$-categories and the $V$-categories of algebras over $V$-triples are constructed in Bunge [1]. Then one can consider

Received 19 June 1970. 
$V$-Trip $(A)$, the category whose objects are the $V$-triples in a $V$-category $A$, and $V-A l g(A)$, the category whose objects are the $V$-categories of $V$-algebras over A.

In this note, using the notion of $V$-limit in the Kelly sense [6] too, we extend in this enriched $v$-context all the results obtained by Frei.

\section{Preliminaries}

We use the notions of symmetric monoidal closed category $V$ and $V$-category, in the Eilenberg-Kelly sense [3]. A $V$-triple $T=(T, n, \mu)$ in a $V$-category $A$ consists of a $V$-functor $T: A \rightarrow A$ and two $V$-natural transformations $n: I_{A} \rightarrow T, \mu: T T \rightarrow T$ so that:

$$
\begin{aligned}
& \text { (i) } \mu \cdot \mu T=\mu \cdot T \mu \text {, and } \\
& \text { (ii) } \mu \cdot T \eta=\mu \cdot \eta T=I_{T} \text {. }
\end{aligned}
$$

A $V$-triple morphism $\tau: T \rightarrow T^{\prime}$ consists of a $V$-natural transformation $\tau: T \rightarrow T^{\prime}$ so that:

(i) $\tau \cdot \eta=\eta^{\prime}$, and

(ii) $\tau \cdot \mu=\mu^{\prime} \cdot \tau T^{\prime} \cdot T \tau$.

Let $A_{0}$ be the underlying category of $A$. A V-triple in $A$ is a triple in $A_{0}$ too. Thus, one can construct the category of algebras $A_{0}^{\top}$, whose objects are pairs $[A, \xi]$ where $A \in A, \xi \in A_{0}(T A, A)$, $\xi \cdot n_{A}=1_{A}, \xi \cdot \mu_{A}=\xi . T \xi$, and whose morphisms are $[f]:[A, \xi] \rightarrow[B, \theta]$ where $f: A \rightarrow B$ in $A_{0}$ and $f . \xi=\theta . T f$. Bunge $([1], 2.2)$ shows that $A_{0}^{T}$ has a $V$-category structure, if $V$ has equalizers (in what follows we suppose this condition to be fulfilled), given by $A^{\top}:\left(A_{0}^{T}\right)^{O p} \times A_{0}^{T} \rightarrow V_{0}$; in this structure $U^{\top}: A_{0}^{\top} \rightarrow A_{0}$, given by $[A, \xi] \mapsto A$ and $[f] \leftrightarrow f$, has a $V$-functor structure and has a $V$-adjoint ${ }_{F}{ }^{\top}$ given at the underlying level by $A \leftrightarrow\left[T A, \mu_{A}\right]$ and $f \leftrightarrow[T f]$. The functor $A^{\top}$ is given on 
objects by $U_{[A, \xi][B, \theta]}^{\top}: A^{\top}([A, \xi][B, \theta]) \rightarrow A(A, B)$, the equalizer of the following pair

$$
A(A, B) \stackrel{\stackrel{T}{\stackrel{{ }_{A B}}{\longrightarrow}} A(T A, T B) \stackrel{A(T A, \theta)}{\longrightarrow}}{\longrightarrow} A(T A, B)
$$

In this way the $V$-category $A^{\top}$ is well defined.

We recall that $R: B \rightarrow A$ is a B-adjoint for $S: A \rightarrow B$ if there exists a V-natural family of isomorphisms $n_{A B}: A(R B, A) \simeq B(B, S A)$ in $v_{0}$, for each $A \in A, B \in B$.

Let $D: K \rightarrow A_{0}$ be a functor and let $B$ be a $V$-category. The family of morphisms $\left\{L \stackrel{\lambda_{K}}{\longrightarrow} D K\right\}_{K \in K}$ in $b_{0}$ is a limit in $B$ (or a $V$-limit) in the Kelly sense [6], if the family $\{B(B, L) \rightarrow B(B, D K)\}_{K \in K}$ is a limit in $V_{0}$, for each $B \in B$.

LEMMA 1.1. If a V-functor $T: A \rightarrow B$ has a V-adjoint $S: B \rightarrow A$ then $T$ preserves the limits of $A$.

Proof. Let $\{L \rightarrow D K\}$ be a limit in $A$. Then $\{A(A, L) \rightarrow A(A, D K)\}$ is a limit in $V_{0}$, by the above definition, for each $A \in A$, and so $\{A(S B, L) \rightarrow A(S B, D K)\}$ are also limits in $V_{0}$, for each $B \in B$. Using the $V$-natural isomorphism $n$, the families $\{B(B, T L) \rightarrow B(B, T D K)\}$ are also limits in $V_{0}$. Hence $\{T L+T D K\}$ is a limit in $B$.

\section{The $V$-isomorphism theorem}

PROPOSITION 2.1. There is a functor $G: V$-Trip $(A) \rightarrow[V-A l g(A)]^{\text {op }}$ given by: $G(T)=A^{\top}$ on objects, and on $\phi: T \rightarrow T^{\prime}$ by a V-functor $G \phi: A^{\top^{\prime}} \rightarrow A^{\top}$, given by $(G \phi)\left[A, \xi^{\prime}\right]=\left[A, \xi^{\prime} \cdot \phi_{A}\right]$ and by a unique morphiam in $v_{0}$

$$
(G \phi)_{\left[A, \xi^{\prime}\right]\left[B, \theta^{\prime}\right]}: A^{T^{\prime}}\left(\left[A, \xi^{\prime}\right]\left[B, \theta^{\prime}\right]\right)+A^{\top}\left(\left[A, \xi^{\prime} \cdot \phi_{A}\right]\left[B, \theta^{\prime} \cdot \phi_{B}\right]\right)
$$


for each $\left[A, \xi^{\prime}\right],\left[B, \theta^{\prime}\right]$ in $A^{T^{\prime}}$.

Proof. Using Diagram 1 on page 379, which commutes, since $U_{\left[A, \xi^{\prime}\right],\left[B, \theta^{\prime}\right]}^{\top^{\prime}}$ is an equalizer and since $\phi$ is $V$-natural, one can find $\mathrm{A}\left(\xi^{\prime} \cdot \phi_{A}, B\right) U_{\left[A, \xi^{\prime}\right]\left[B, \theta^{\prime}\right]}^{\mathrm{T}^{\prime}}=\mathrm{A}\left(T A, \theta^{\prime} \cdot \phi_{B}\right) \cdot T_{A B} \cdot U_{\left[A, \xi^{\prime}\right]\left[B, \theta^{\prime}\right]}^{T^{\prime}} \cdot$

$U^{\top}\left[A, \xi^{\prime} \cdot \phi_{A}\right]\left[B, \theta^{\prime} \cdot \phi_{B}\right]$ being an equalizer it follows that there exists a unique morphism $(G \phi)\left[A, \xi^{\prime}\right]\left[B, \theta^{\prime}\right]$ so that

$$
U_{\left[A, \xi^{\prime}\right]\left[B, \theta^{\prime}\right]}^{T^{\prime}}=U_{\left[A, \xi^{\prime} \cdot \phi_{A}\right]\left[B, \theta^{\prime} \cdot \phi_{B}\right]}^{\top} \cdot(G \phi)_{\left[A, \xi^{\prime}\right]\left[B, \theta^{\prime}\right]} \cdot
$$

As in [1], the $G \phi^{\prime} s$ defining equality taking place also at the underlying level, it follows that $G \phi$ is a $V$-functor. Being compatible with the forgetful functors, $G \phi$ is a morphism in $[U-A l g(A)]^{o p}$.

PROPOSITION 2.2. There is a functor $H:[V-A I g(A)]^{\mathrm{OP}}+V-\operatorname{Trip}(A)$ given by $H\left(A^{\top}\right)=\mathrm{T}$ and by $H(R): \mathrm{T} \rightarrow \mathrm{T}^{\prime}$, a V-natural transformation given in $A_{0}$ by the fomizy, $H(R)_{A}^{0}=U_{0} a_{A, R F^{\prime} A}^{-1}\left(\eta_{A}^{\prime}\right)$ where $U_{0}^{\top}: A_{0}^{\top} \rightarrow A_{0}$, $a_{A, R F^{\prime} A}^{-1}: \mathrm{A}_{0}\left(A, T_{0}^{\prime} A\right) \approx A_{0}^{\top}\left(F A, R F^{\prime} A\right)$ are the underlying corresponding notions and we denote $F^{\top}$ and $F^{\top}$ simply by $F, F^{\prime}$.

Proof. According to [5] it remains to show that $H(R)$ is V-natural. Using the formula $a_{X,[Y, \sigma]}^{-1}(g)=[\sigma . T g]$ for $g: X \rightarrow U^{\top}([Y, \sigma])=Y$ and by the remark $R\left(\left[X, \xi^{\prime}\right]\right)=\left[X, R\left(\xi^{\prime}\right)\right]$ one can show that $H(R)_{A}^{\circ}=R_{0}\left(\mu_{A}^{\prime}\right) \cdot T_{0} \eta_{A}^{\prime}$. The V-naturality of $H(R)$ follows now from the $V$-naturality of $\eta^{\prime}$ and $\mu^{\prime}$ and from the $V$-functoriality of $R$ and $T$ ([3], I, 10.2).

THEOREM 2.3. The functors $G$ and $H$ are inverse to each other, that is, $\quad$-Trip $(A)$ is isomorphic to $\left[V_{-A l g}(A)\right]^{o p}$.

Proof. As in [5]. 
Triples in enriched categories

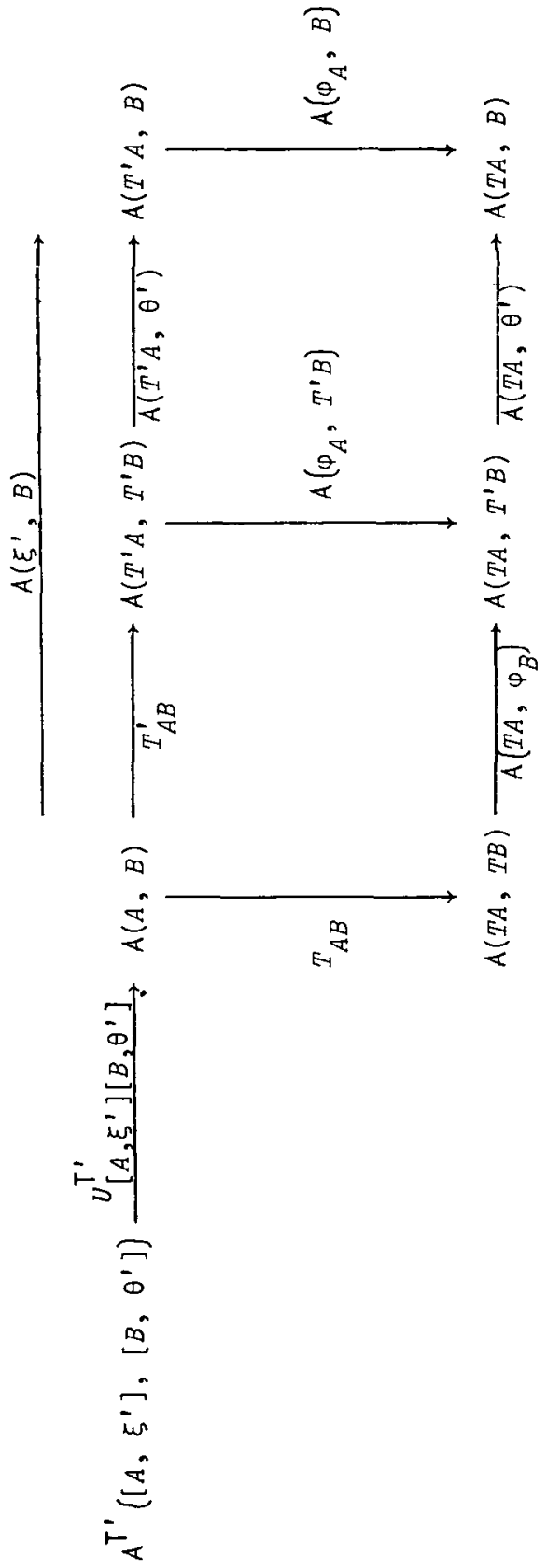

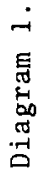




\section{The morphisms of $V-A \lg (A)$}

PROPOSITION 3.1. Let $T$ be a V-triple in $A$ and $U: A^{\top} \rightarrow A$ be the corresponding underlying $V$-junctor. Then a V-functor $G: B+A^{T}$ preserves limits in $B$ iff $U G$ does.

Proof. Using Lema 1.1, the condition is easily seen to be necessary, $F^{\top}: A \rightarrow A^{\top}$ being a $V$-adjoint for $U$. Conversely, let $W$ be an object in $v_{0},[A, \xi]$ an object in $A_{0}^{\top}$ and $\alpha_{K}: W \rightarrow A^{\top}([A, \xi], G K), K \in K$ a compatible (in $K$ ) family of morphisms in $V_{0}$. We show that there exists a unique morphism $f: W \rightarrow A^{\top}([A, \xi], G L)$ so that $\alpha_{K}=A^{\top}\left([A, \mu], G_{0} \lambda_{K}\right) \cdot f$.

In Diagram 2, on page 381 , all the squares commute, since $A(T A,-)$ is a functor, $G_{0} \lambda_{K}$ is an algebra morphism and since $\theta$ : TUGL $\rightarrow$ UGL and $\theta_{K}: T U G D K \rightarrow U G D K$ are the T-structures of $U G L$ and $U G D K$. Since $U G$ preserves the limits of $A$, for each $A \in A$, $A(A, U G L) \stackrel{A\left(1, U_{0} G_{0} \lambda_{K}\right)}{\longrightarrow} A(A, U G D K)$ is a limit in $v_{0}$. Since $\alpha_{K}$ is a compatible family, there exists a unique morphism $g: W \rightarrow \mathrm{A}(A, U G L)$ so that $U \cdot \alpha_{K}=A\left(\dot{I}, U_{O} G_{0} \lambda_{K}\right) \cdot g$ and hence we have another commuting square in Diagram 2 .

Now, we show that $g$ equalizes the pair $A(T A, \theta) . T$ and $A(\xi, U G L)$. Indeed we have $A\left(1, U G \lambda_{K}\right) \cdot A(\xi, U G L) \cdot g=A(\xi, U G D K) \cdot A\left(1, U G \lambda_{K}\right) \cdot g=A(\xi, U G D K) \cdot U \cdot \alpha_{K}=$
$A\left(T A, \theta_{K}\right) \cdot T \cdot U \cdot \alpha_{K}=A\left(T A, \theta_{K}\right) \cdot T \cdot A\left(1, U G \lambda_{K}\right) \cdot g=A\left(1, U G \lambda_{K}\right) \cdot A(T A, \theta) \cdot T \cdot g$, and so the above statement follows from the fact that $A\left(l, U G \lambda_{K}\right)$ are limits in $V_{0}$. Hence there exists a unique morphism $f: W \rightarrow A^{\top}([A, \xi], G L)$ so that $g=U \cdot f$. But,

$$
U \cdot \alpha_{K}=A\left(1, U G \lambda_{K}\right) \cdot g=A\left(1, U G \lambda_{K}\right) \cdot U \cdot f=U A^{\top}\left(1, G_{0} \lambda_{K}\right) \cdot f,
$$




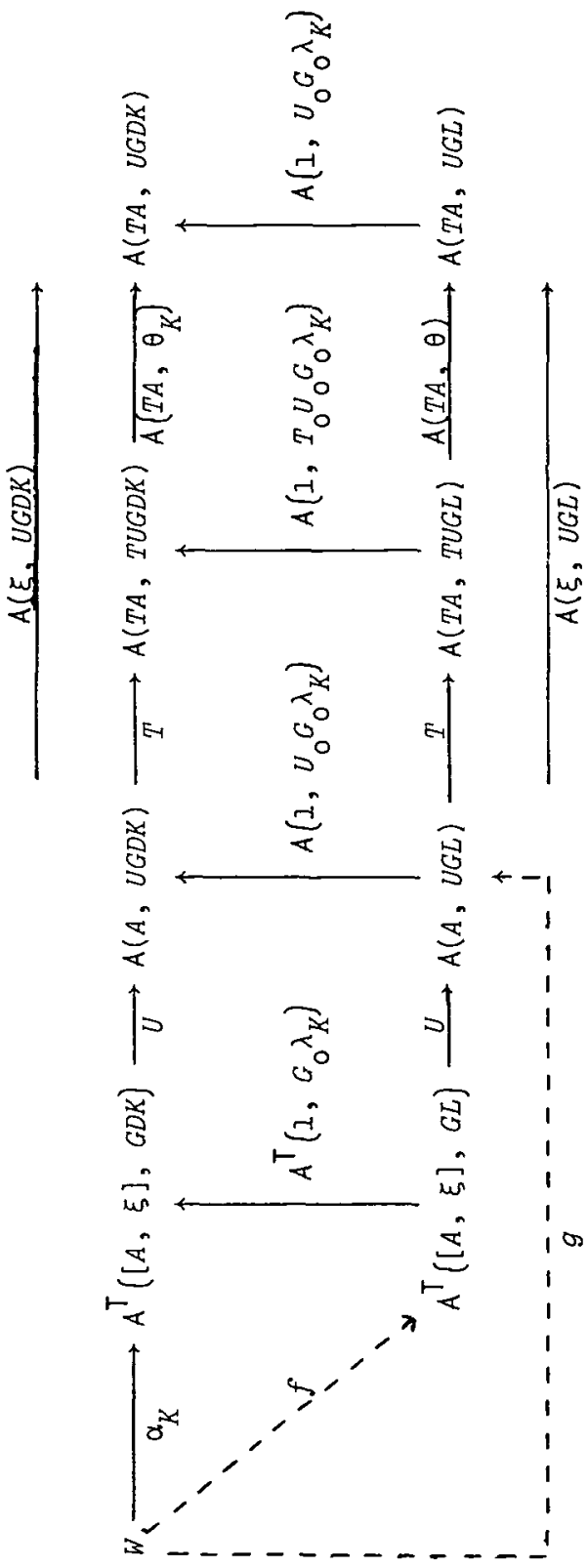

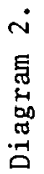


and so, $U$ being monic, the triangle in Diagram 2 commutes. In this way we already have the existence of the morphism $W+A^{\top}([A, \xi], G L)$ we were looking for.

The uniqueness follows from the fact that $g$ is unique for its defining commutation and from the fact that if $f \neq f^{\prime}$ then $U f \neq U f^{\prime}$ and hence $A\left(1, U G \lambda_{K}\right) \cdot U \cdot f \neq A\left(1, U G \lambda_{K}\right) \cdot U \cdot f^{\prime}$, the motivation of this last fact being already mentioned above.

The theorem and corollary which follow are immediate consequences of the last proposition.

THEOREM 3.2. The morphisms in $V-A I g(A)$ preserve limits.

COROLLARY 3.3. Let $T$ be a V-triple in A, $(\gamma, \varepsilon): S+T: B \rightarrow A$ a V-adjoint pair which generates $T$, and $L: B \rightarrow A^{\top}$, the canonical V-functor given by $L B=\left[T B, T \Upsilon_{B}\right]$ and by $L_{B B^{\prime}}$, as in 2.3 in [1], $L$ preserves limits.

\section{References}

[1] Marta C. Bunge, "Relative functor categories and categories of algebras", J. AZgebra 11 (1969), 64-101.

[2] B.J. Day and G.M. Kelly, "Enriched functor categories", Reports on the Midwest Category Seminar III (Lecture notes in Mathematics, 106, 178-191. Springer-Verlag, Berlin, Heidelberg, New York, 1969).

[3] Samuel Ei lenberg and G. Max Kelly, "Closed categories", Proc. Conf. Categorical Algebra (La Jolla, Calif., 1965), 421-562. (Springer-Verlag, Berlin, Heidelberg, New York, 1966).

[4] Samuel Eilenberg and John C. Moore, "Adjoint functors and triples", IZlinois J. Math. 9 (1965), 381-398.

[5] A. Frei, "Some remarks on triples", Math. Z. 109 (1969), 269-272. 
[6] G.M. Kelly, "Adjunction for enriched categories", Reports on the Midwest Category Seminor III (Lecture notes in Mathematics, 106, 166-177. Springer-Verlag, Berlin, Heidelberg, New York, 1969).

University of Cluj,

Cluj, Roumania. 